\title{
Activin-A exerts a crucial anti-inflammatory role in neonatal infections
}

\author{
Eutichia Petrakou', Spyros Fotopoulos', Marina Anagnostakou', Fani Anatolitou', Konstantinos Samitas², Maria Semitekolou², \\ Georgina Xanthou' and Marietta Xanthou ${ }^{1,3}$
}

BACKGROUND: Activin-A is a cytokine with a critical role in infections and associated inflammation in experimental models and humans. Still, the effects of activin-A on neonatal infections remain elusive. Here, we investigated the expression of activin-A in the serum of septicemic preterm and term neonates and in peripheral blood leukocytes stimulated with inflammatory agents in vitro. The role of activin-A in the regulation of inflammatory responses by neonatal leukocytes was delineated.

METHODS: Peripheral blood was obtained from 37 septicemic neonates between the first and fifth days postinfection and from 35 healthy controls. Isolated monocytes and lymphocytes were stimulated with lipopolysaccharide (LPS) or phytohemagglutinin (PHA) in vitro in the presence of activinA. Cell proliferation, cytokine, and chemokine release were investigated.

RESULTS: Activin-A was significantly increased in the serum of preterm septicemic neonates. Neonatal leukocytes secreted copious amounts of activin-A following stimulation, pointing to these cells as an essential source of activin-A in the circulation. Of note, treatment of neonatal leukocytes with activinA during PHA and LPS stimulation resulted in significantly decreased interleukin (IL)-1 $\beta, \quad \mid \mathrm{L}-6$, and CXCL8 production, concomitant with a striking increase in the anti-inflammatory mediator, IL-10.

CONCLUSION: Our findings uncover activin-A as a novel immunomodulatory agent critical for the control of inflammatory responses in septicemic neonates.

D uring infections, there is enhanced release of pro- and anti-inflammatory mediators, such as cytokines and chemokines that play a central role in the neonatal host defense against pathogens (1). Proinflammatory mediators activate protective immune responses toward infectious agents, whereas anti-inflammatory factors suppress immune cell activation and promote tissue repair. As exuberant inflammatory responses against infectious stimuli in neonates can lead to tissue cell death and organ damage, the expression and activation of anti-inflammatory cytokines is vital for the maintenance of neonatal tissue homeostasis $(2,3)$.
Activin-A is a pleiotropic cytokine that belongs to the transforming growth factor- $\beta 1$ superfamily and plays a crucial role in the fundamental biological processes, such as development, stem cell pluripotency, and tissue repair $(4,5)$. Growing evidence suggests that activin-A is also induced during inflammatory processes and, more specifically, during infections (68). For example, administration of bacterial endotoxin (LPS) in vivo induces a rapid (within $40 \mathrm{~min}$ ) release of activin- $\mathrm{A}$ in the circulation (7). Of note, activin-A expression is observed at an earlier time point as compared with that of tumor necrosis factor (TNF)- $\alpha$ and well ahead the release of IL-6 $(7,8)$. In addition, activin-A levels are significantly increased following in vitro stimulation with toll-like receptor ligands (including viruses and bacteria) or proinflammatory cytokines (i.e., TNF- $\alpha$ and IFN- $\gamma$ ) in a wide array of cells, such as monocytes, dendritic cells (DCs), lymphocytes, epithelial, and endothelial cells $(4,5)$.

There are only few studies that have investigated activinA expression in the perinatal and the neonatal periods. Increased activin-A levels have been observed in the placenta and in the maternal and fetal serum of intrauterine growth retardation pregnancies $(9,10)$. Furthermore, Florio et al. have demonstrated significantly increased activin-A in preterm newborns with signs of perinatal hypoxia at birth (11). Activin-A was also upregulated in the cerebrospinal fluid of asphyxiated full-term newborns (12). In fact, growing evidence proposes that activin-A should be used as a biomarker for hypoxic-ischemic encephalopathy in asphyxiated neonates $(13,14)$. Activin-A is significantly increased in the amniotic fluid in women with premature rupture of the membranes and infection-induced preterm birth (15). Still, the effects of activin-A on neonatal infections and associated inflammation are elusive.

In this study, we investigated the expression of activin-A in the serum of preterm and term neonates with septicemia and in the neonatal peripheral blood lymphocytes and monocytes following stimulation with inflammatory mediators in vitro. In addition, the effects of activin-A on inflammatory responses mediated by neonatal peripheral blood leukocytes were examined. 


\section{RESULTS}

Increased Activin-A Levels in Preterm Neonates During Infections Initially, we investigated the expression of activin-A in the serum of neonates with septicemia. Activin-A levels were significantly increased in neonates with birth weight $(\mathrm{BW})<$ $1,500 \mathrm{~g}$ on the first $(P<0.05)$, third $(P<0.05)$, and fifth $(P<0.05)$ days postinfection, as compared with those of healthy controls (Figure 1a). Septicemic neonates with BW =1,500-2,800 g had also significantly increased activin-A levels in the serum on the third and fifth days ( $P<0.05$ and $P<0.05$, respectively), as compared with controls (Figure 1b). Activin-A serum levels in infected full-term neonates were increased; however, they did not reach statistical significance (Figure 1c).

We next performed time-course studies to determine the kinetics of activin-A expression in the serum of preterm septicemic neonates. Serum levels of the well-known proinflammatory chemokine, CXCL8, were also examined and compared with those of activin-A. Our data demonstrated that activin-A expression gradually increased in the serum of septicemic preterm neonates ( $\mathrm{BW}=1,500-2,800 \mathrm{~g}$ ), as shown by significantly elevated levels on the third and fifth days postinfection, as compared with the first day $(P<0.05$ and $P<0.01$, respectively) (Figure 2a). Activin-A levels were not significantly different between the third and fifth days postinfection. In sharp contrast, the expression of CXCL8 gradually decreased in the serum of septicemic preterm neonates ( $\mathrm{BW}=1,500-2,800 \mathrm{~g})$, as demonstrated by significantly decreased levels on the fifth $(P<0.001)$ day, as compared with the first day. CXCL8 levels were also decreased between the third and fifth days postinfection but did not reach statistical significance (Figure 2b). Furthermore, two-way ANOVA revealed a statistical association between activin-A and CXCL8 serum levels (data not shown). In fact, a strong inverse correlation was observed between activin-A and CXCL8 ( $P=0.004 ; r=-0.7039$; Figure $2 \mathrm{c}$ ). Pertinent to the group of neonates with $\mathrm{BW}<1,500 \mathrm{~g}$, our results showed that activin-A and CXCL8 also exhibited distinct kinetic patterns with a gradual increase in activin-A and a decrease in CXCL8 (activin-A levels: $365.8 \pm 36.9 \mathrm{pg} / \mathrm{ml}$ on d 1, 394.8 $\pm 33.2 \mathrm{pg} / \mathrm{ml}$ on $\mathrm{d} 3,414.8 \pm 36.9 \mathrm{pg} / \mathrm{ml}$ on $\mathrm{d} 5$; CXCL8 levels: $386.3 \pm 15.2$ $\mathrm{pg} / \mathrm{ml}$ on $\mathrm{d} 1,188.4 \pm 21.6 \mathrm{pg} / \mathrm{ml}$ on $\mathrm{d} 3,170.5 \pm 21.1 \mathrm{pg} / \mathrm{ml}$ on a

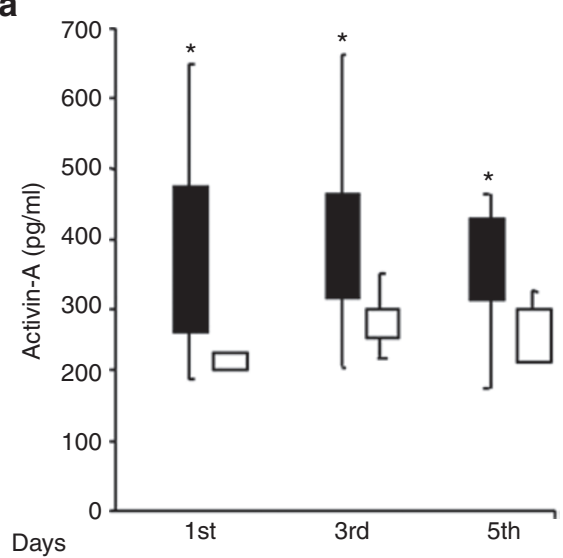

b

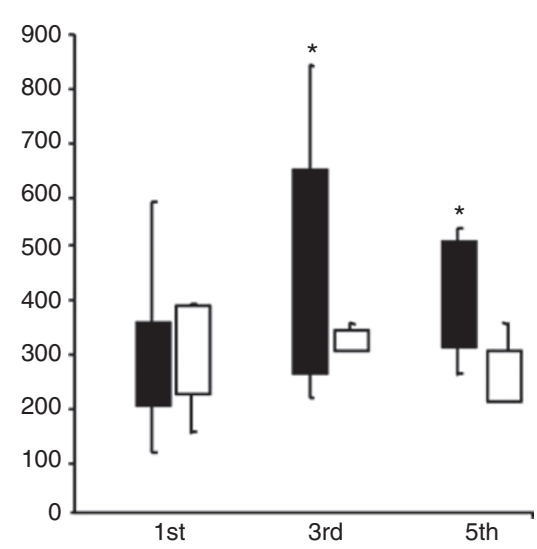

C

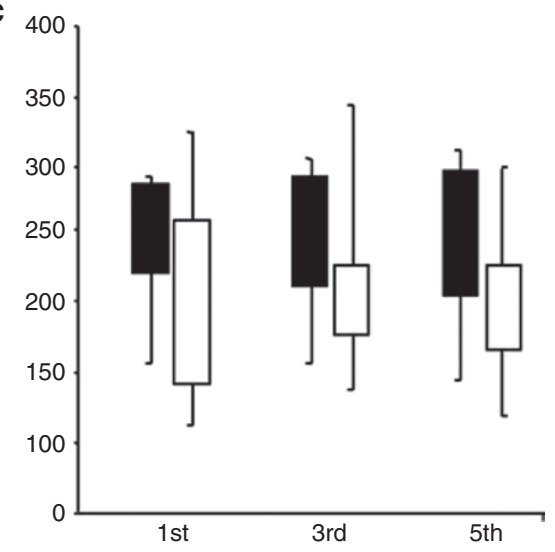

Figure 1. Activin-A is increased in the serum of septicemic neonates. Activin-A levels in the serum of septicemic and healthy neonates with (a) birth weight (BW) $<1,500 \mathrm{~g}$, (b) BW =1,500-2,800 g, and (c) BW $>2,800 \mathrm{~g}$. (a) Activin-A levels are significantly elevated in the serum of neonates with nosocomial infections (black fill, $n=16$ ) on the first, third, and fifth days, as compared with healthy controls (white fill, $n=16$ ). (b) Septicemic neonates with $\mathrm{BW}=1,500-2,800 \mathrm{~g}$ (black fill, $n=11$ ) have significantly increased activin-A levels in the serum on the third and fifth days postinfection, as compared with controls (white fill, $n=11$ ). (c) Activin-A levels are upregulated in septicemic full-term neonates (black fill, $n=10$ ), as compared with healthy controls (white fill, $n=8$ ); however, no statistical significance is observed. Data are presented as mean $\pm \mathrm{SD}, * P<0.05$.
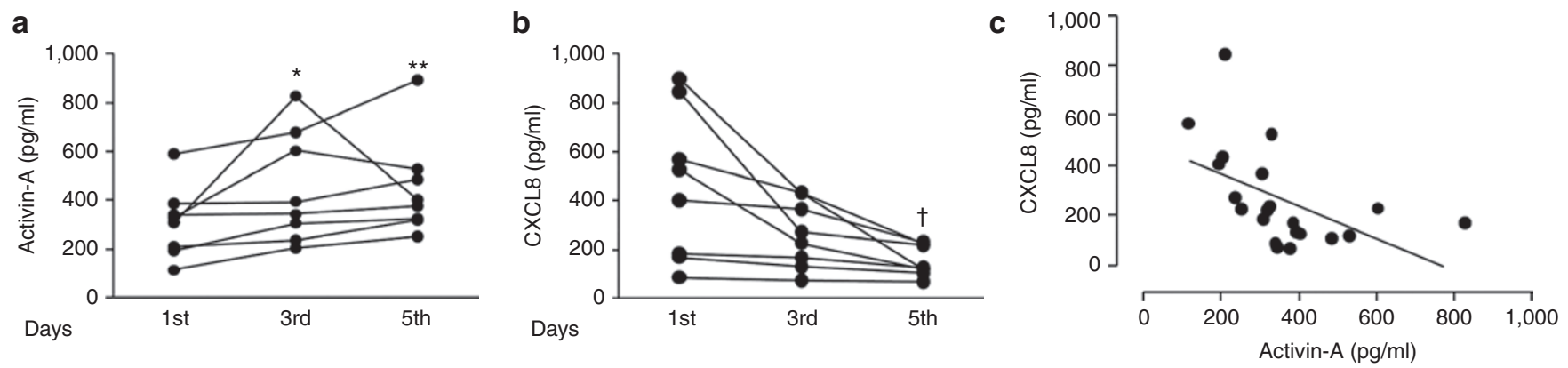

Figure 2. CXCL8 and activin-A exhibit distinct expression patterns in the serum of septicemic neonates. (a) Activin-A levels are significantly increased in the serum of septicemic preterm neonates (birth weight $(B W)=1,500-2,800 \mathrm{~g}$ ) on the third and fifth days postinfection, as compared with the first day. (b) CXCL8 serum levels in septicemic preterm neonates ( $B W=1,500-2,800 \mathrm{~g})$ are significantly decreased on the fifth day, as compared with the first day. CXCL8 levels are also decreased, but not significantly, between the third and fifth days postinfection. (c) An inverse correlation was observed between the serum levels of activin-A and CXCL8 $(P=0.004 ; r=-0.7039)$. Data shown are from $n=8$ neonates, ${ }^{*} P<0.05,{ }^{* *} P<0.01$, and ${ }^{\dagger} P<0.001$. 
d 5). However, the data reached statistical significance only for CXCL8 ( $P=0.0273$ using Friedman test; post hoc analysis showed no statistical significance between groups). In addition, there was no significant correlation between activin-A and CXCL8 expression $(P=0.263 ; r=-0.18)$. Overall, our data uncover activin-A as a novel mediator involved in neonatal infections.

\section{Activin-A Expression Is Upregulated in Neonatal Peripheral Blood Mononuclear Cells Following Stimulation In Vitro}

We subsequently investigated the ability of neonatal peripheral blood mononuclear cells (PBMCs) to produce activin-A following exposure to inflammatory stimuli in vitro. Activin-A was expressed at low levels in nonstimulated peripheral blood lymphocytes and monocytes of healthy neonates (Figure $3 a, b)$. Of note, activin-A was dramatically (more than 10-fold) increased in neonatal peripheral blood lymphocytes following stimulation with the mitogen phytohemagglutinin (PHA) in vitro, as compared with nonstimulated cells. Activin-A levels were increased at all time points studied and reached statistical
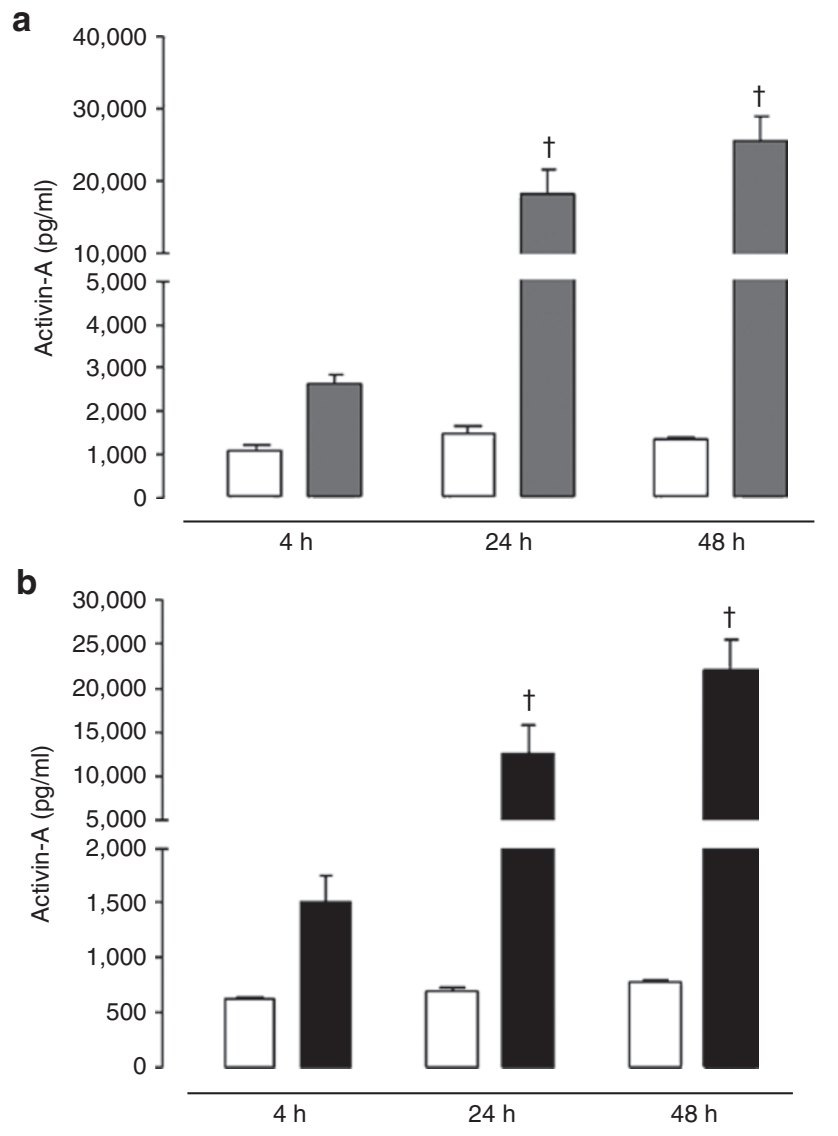

Figure 3. Activin-A levels following stimulation of neonatal peripheral blood mononuclear cells in vitro. (a) Activin-A release is increased in neonatal peripheral blood lymphocytes following in vitro stimulation with phytohemagglutinin (gray fill) at all time points studied, achieving statistical significance at 24 and $48 \mathrm{~h}$, as compared with nonstimulated cells (white fill, $n=10$ healthy neonates). (b) Activin-A levels are also significantly upregulated in neonatal peripheral blood monocytes upon in vitro stimulation with lipopolysaccharide (black fill, $n=9$ healthy neonates) at the 24and 48 -h time points. Data are presented as mean \pm SEM, ${ }^{+} P<0.001$. significance at $24 \mathrm{~h}(P<0.001)$ and $48 \mathrm{~h}(P<0.001)$ (Figure 3a). Activin-A levels in PHA-stimulated lymphocytes reached a peak at $48 \mathrm{~h}$ (Figure $3 \mathrm{a}$ ).

Similarly, in vitro stimulation of neonatal peripheral blood monocytes with LPS, resulted in a significant upregulation in activin-A production at both $24 \mathrm{~h}(P<0.001)$ and $48 \mathrm{~h}(P<$ 0.001 ), as compared with nonstimulated monocytes (Figure $3 b)$. The release of activin-A by neonatal monocytes reached a maximum at $48 \mathrm{~h}$ following LPS stimulation (Figure $3 \mathrm{~b}$ ). Overall, our findings demonstrate that activation of neonatal peripheral blood lymphocytes and monocytes with inflammatory stimuli induces a robust release of activin-A, supporting a role for this cytokine in the regulation of neonatal immune responses.

\section{Activin-A Suppresses Inflammatory Cytokine and Chemokine Release by Neonatal PBMCs}

Next, studies were undertaken to elucidate the role of activinA in inflammatory responses mediated by neonatal PBMCs. For this, we isolated peripheral blood lymphocytes and treated them with $\mathrm{r}$-activin-A (or phosphate-buffered saline control) during in vitro stimulation with the strong T-cell mitogen, PHA (16-18). Initially, our data showed that treatment of neonatal peripheral blood lymphocytes with PHA, resulted in significantly increased production of IL- $1 \beta(P<0.01)$, IL-6 $(P<0.01)$, CXCL8 $(P<0.01)$, and IL-10 $(P<0.01)$, as compared with nonstimulated cells (Figure $4 a-d)$. Of note, $r$-activin-A induced a significant decrease in PHA-induced release of IL-1 $\beta(P<0.05)$ and CXCL8 $(P<0.01)$ in the supernatants of peripheral blood lymphocytes (Figure $4 \mathrm{a}-\mathrm{c}$ ). Of note, CXCL8 levels in activinA-treated lymphocytes reached levels similar to those of nonstimulated control cells. IL-6 levels were also decreased following r-activin-A treatment compared with PHA-treated cells; however, they did not reach statistical significance (Figure $4 \mathrm{~b}$ ). By contrast, treatment with $r$-activin-A resulted in a significant increase in the levels of the anti-inflammatory cytokine IL-10 $(P<0.01)$, as compared with PHA-treated cells (Figure $4 \mathrm{~d}$ ). TNF- $\alpha$ levels were not significantly different between PHA and activin-A/PHA-treated lymphocytes (data not shown). Of note, activin-A did not affect lymphocyte viability or proliferation, as examined by the 3-4,5-dimethylthiazol-2-yl-2,5 diphenyl tetrazolium bromide assay (data not shown).

We subsequently investigated the effects of activin-A in LPS-induced responses by neonatal peripheral blood monocytes that express enhanced levels of the LPS receptor, toll-like receptor-4, and respond highly to this stimulus $(1,19-21)$. Our findings demonstrated that LPS induced a dramatic increase in the production of IL- $1 \beta(P<0.01)$, IL-6 $(P<0.01)$, CXCL8 $(P<0.01)$, and IL-10 $(P<0.01)$ by neonatal peripheral blood monocytes, as compared with nonstimulated cells (Figure $5 \mathrm{a}-\mathrm{d})$. Similar to what we observed in the responses mediated by neonatal lymphocytes, $\mathrm{r}$-activin-A treatment resulted in significantly decreased LPS-induced release of IL-1 $\beta(P<$ $0.01)$, IL-6 $(P<0.01)$, and CXCL8 $(P<0.01)$ in culture supernatants of neonatal peripheral blood monocytes (Figure $5 \mathrm{a}-\mathrm{c}$ ). Stimulation of peripheral blood monocytes with LPS resulted 


\section{Articles | Petrakou et al.}
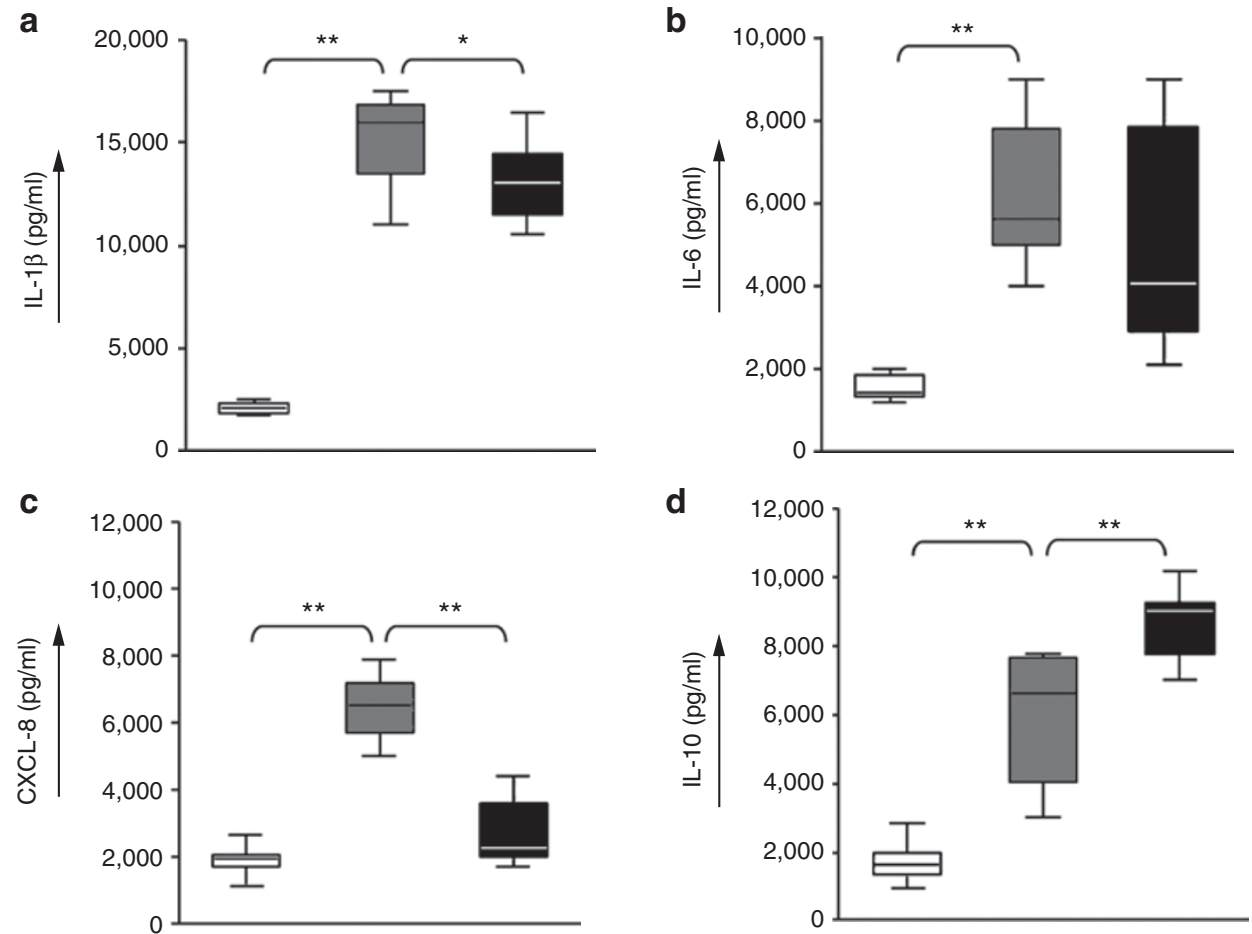

Figure 4. Activin-A effects on phytohemagglutinin (PHA)-induced inflammatory responses by neonatal peripheral blood lymphocytes. Treatment of neonatal lymphocytes with PHA (gray fill) results in significantly increased production of (a) IL-1 $\beta$, (b) IL-6, (c) CXCL8, and (d) IL-10 as compared with nonstimulated cells (white fill). Activin-A treatment (black fill) significantly decreases PHA-driven release of (a) IL-1 $\beta$ and (c) CXCL8 in culture supernatants of neonatal lymphocytes. (d) Treatment with r-activin-A (black fill) significantly increases IL-10 levels, as compared with PHA-treated lymphocytes (gray fill). Data are presented as mean $\pm \mathrm{SEM}, n=10$ healthy neonates, ${ }^{*} P<0.05,{ }^{* *} P<0.01$.
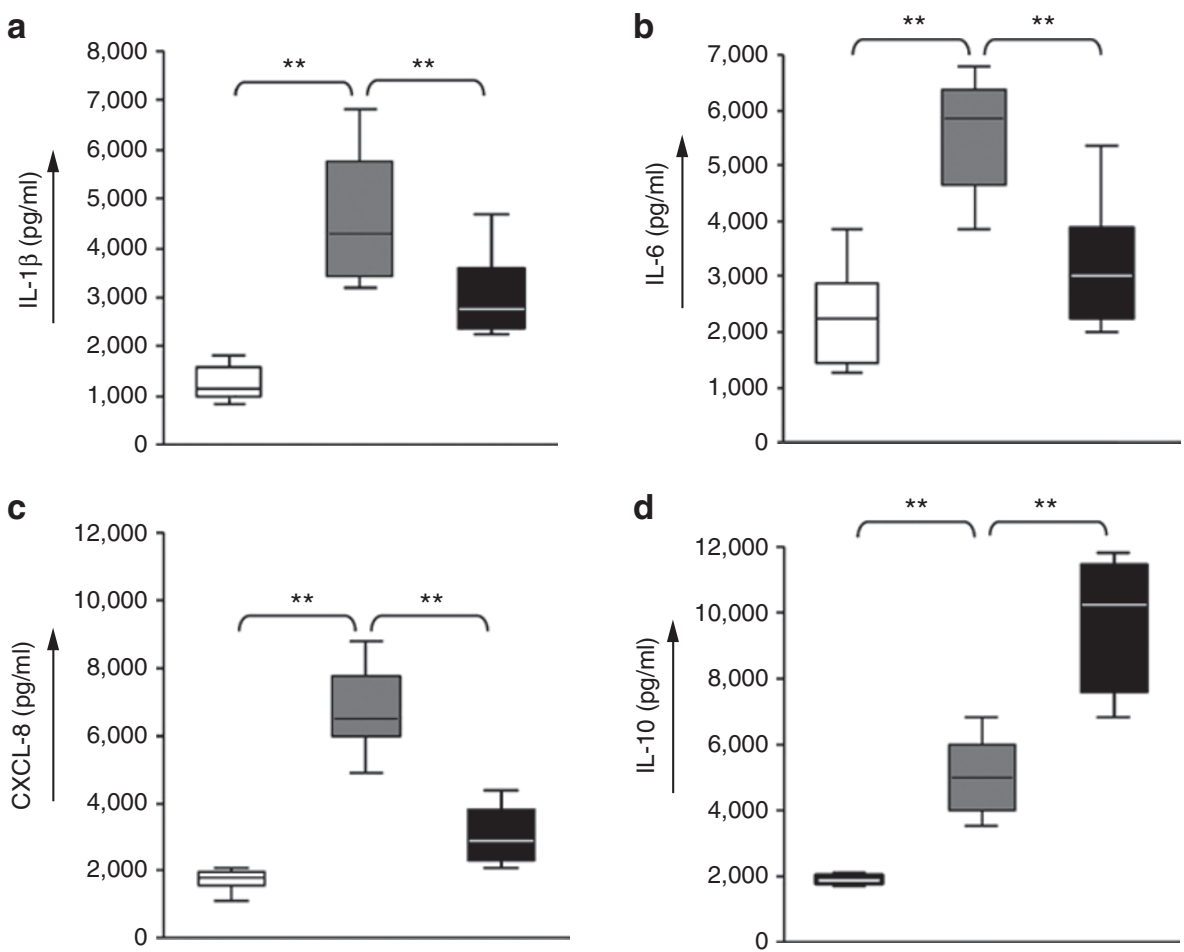

Figure 5. Activin-A effects on lipopolysaccharide (LPS)-mediated inflammatory cytokine release by neonatal peripheral blood monocytes.Treatment of neonatal monocytes with LPS (gray fill) induces a significant increase in the production of (a) IL-1 $\beta$, (b) IL-6, (c) CXCL8, and (d) IL-10, as compared with nonstimulated cells (white fill). Activin-A treatment (black fill) induces a significant decrease in LPS-induced release of (a) IL-1 $\beta$, (b) IL-6, and (c) CXCL8 by neonatal peripheral blood monocytes. (d) IL-10 levels are significantly elevated in culture supernatants following treatment with $\mathrm{r}$-activin-A (black fill), as compared with LPS-treated lymphocytes (gray fill). Data are presented as mean $\pm \mathrm{SEM}, n=10$ healthy neonates, ${ }^{* *} P<0.01$. 
in enhanced release of TNF- $\alpha$ compared with nonstimulated cells. TNF- $\alpha$ levels were decreased following $r$-activin-A treatment; however, the data did not reach statistical significance (data not shown). Of note, peripheral blood monocytes produced copious amounts of IL-10 following treatment with r-activin-A $(P<0.01)$ as compared with LPS-treated cells (Figure 5d). Collectively, our data reveal that activin-A can restrain robust immune responses generated by neonatal PBMCs during stimulation with inflammatory agents in vitro.

\section{DISCUSSION}

The findings of this study uncover activin-A as a novel critical controller of inflammatory responses in septicemic neonates. Although previous studies have demonstrated induction of activin-A during infections in animal models and in adults $(6-8,22,23)$, as far as we know, this is the first study that investigated activin-A expression in neonates. We demonstrate that activin-A is significantly increased in the serum of neonates during infections. Of note, activin-A levels are greatly upregulated in low BW septicemic neonates and decrease as the gestational age (GA) increases. The increase in activin-A expression in the serum of low BW neonates could be due to the activation of an endogenous protective mechanism to control excessive inflammatory responses often observed in this cohort $(2,24,25)$. In support of this notion, our studies reveal that activin-A can significantly suppress the release of proinflammatory mediators by neonatal PBMCs during in vitro stimulation with LPS and PHA. Of note, activin-A inhibitory effects are accompanied by a dramatic increase in the production of the anti-inflammatory mediator, IL-10. These data support a, newly identified, link between activin-A and IL-10 in the regulation of immune responses in neonates.

Comparing the kinetics of activin-A's expression in the serum during neonatal infections with those of CXCL8, we observed that there was an inverse correlation between activin-A and CXCL8 levels, pointing to a crossregulation between the two factors. The increase in activin-A expression at a late time point in septicemic neonates could be part of a negative feedback circuit aiming to control the earlier release of CXCL8 and other proinflammatory mediators. In support of this notion, activin$\mathrm{A}$ has been shown to induce a dose-dependent decrease in CXCL8 release in PBMCs from angina patients (26). A similar pattern of expression during infections has been observed for other immunomodulatory cytokines, such as IL-10 and transforming growth factor- $\beta_{1}$ (27). In agreement with our findings, other studies have demonstrated that activin-A is elevated in the circulation during septicemia in adults and associated with the severity of inflammation (28). Moreover, activin-A is increased in the cerebrospinal fluid during meningitis in animal models and adults $(22,23)$. Serum levels of activin-A vary with certain studies reporting high levels $(11,29)$ and other studies reporting similar levels, or even, reporting lower levels to our study $(9,28,30,31)$. This discordance could be due to the differences in the experimental set up, including the age of the neonates studied, the disease context, the methodology employed, the time point of analysis, etc. It is also conceivable that activin-A levels are different in neonates with asphyxia/ hypoxia, as a result of the distinct inflammatory response that occurs as compared with infection.

Our studies also show that activated PBMCs are an essential source of activin-A in the circulation during neonatal infections. In support, studies in experimental models and adults have shown that activin- $\mathrm{A}$ is increased in monocytes and DCs upon stimulation with infectious agents and inflammatory cytokines in vitro and in vivo $(4,5,32)$. Nevertheless, a possible role for endothelial and/or other immune cells in the release of activin-A during neonatal infections cannot be excluded. In fact, a recent study demonstrated that circulating neutrophils release high levels of activin-A upon stimulation with TNF- $a$ exvivo (33).

Pertinent to the effects of activin-A on neonatal immune responses, we demonstrate that activin-A suppresses the release of CXCL-8, IL-6, and IL- $1 \beta$ by neonatal PBMCs, while inducing a striking increase in the production of IL-10. Still, TNF- $\alpha$ release remained unchanged following treatment with activin-A. Certain studies have demonstrated that activinA enhances TNF- $\alpha$ release (34), whereas others have shown inhibitory effects (32). We postulate that TNF- $\alpha$ levels may vary depending on the experimental protocol used, including, among others, the time point of analysis and the concentration of LPS. In agreement with our findings, studies by other investigators and our group have shown that activin-A increases IL-10 expression in mouse CD4 $4^{+} \mathrm{T}$ cells (35) and human DCs (32). Moreover, neutralization of IL-10 reverses activin-Amediated suppression of mouse T-cell responses, pointing to IL-10 as an essential mediator of activin-A's functions (35). Considering that there is a striking increase in activin-A levels in preterm neonates during infection in vivo, it will be interesting to examine whether this newly identified link between activin-A and IL-10 is also applicable in PBMCs from preterm neonates.

Studies in other settings have also demonstrated anti-inflammatory effects for activin-A. For example, a strong neuroprotective role for activin- $\mathrm{A}$ in acute brain damage associated with decreased inflammation and increased neuron regeneration has been observed (36). In addition, treatment of human DCs with activin-A's inhibitor, follistatin, greatly enhances CD40 ligand-induced inflammatory cytokine and chemokine release (32). As DCs produce activin-A in inflammatory settings, these findings propose an autocrine regulatory role for activin-A in controlling DC responses. Of note, activin-A induces the generation of mouse regulatory $\mathrm{T}$ cells suppressive against responses by other $\mathrm{T}$ cells in vitro and in vivo $(5,35)$. By contrast, certain studies point to proinflammatory effects of activin-A as shown by enhanced survival during systemic administration of follistatin following a lethal dose of LPS in vivo (8). Moreover, increased TNF- $\alpha$ and IL- $1 \beta$ release was observed upon stimulation of rat macrophages with activinA in vitro (42). We believe that the discordance between the results presented in the latter study and ours may be due to the differences in the experimental set up employed (i.e., distinct species and cell types, stimulation protocol, and time point of 
analysis). Notwithstanding this, our studies provide further support for the concept that activin-A can exert both enhancing and inhibitory effects on inflammatory cytokine release depending on the cell type, the existing cytokine milieu, and the context of the immune response $(4,5)$.

In conclusion, our findings highlight a strong anti-inflammatory role for activin-A in neonatal infections. In fact, our functional analyses propose that activin-A is increased in the serum of septicemic neonates to suppress excessive cytokine and chemokine release and to prevent overt inflammation and tissue damage. Our findings have important clinical implications for the use of activin-A as a new therapeutic target in neonatal infection and inflammation.

\section{METHODS}

\section{Subjects}

Neonates with septicemia ( $n=37,20$ male, 17 female) were classified into three groups according to their BW and GA: group A (BW $<1,500$ g; GA: $28 \mathrm{wk}(24-33), n=16)$, group B (BW = 1,500-2,800 g; GA: $34 \mathrm{wk}(28-38), n=11)$, and group C (BW > 2,800 g; GA: $38 \mathrm{wk}$ (37-41), $n=10)$. Neonates ( $n=35,19$ male, 16 female) with no sign of infection or asphyxia/hypoxia, no need for supplementary oxygen or ventilator use, and no sign of intraventricular hemorrhage were considered as healthy controls. These healthy controls and neonates with septicemia were matched for GA, BW, and postnatal age. Regarding the neonates' age, samples were collected at $12 \pm 2.4$ postnatal days for the septic groups and $11 \pm 2.1$ postnatal days for the healthy controls (mean \pm SEM). Sepsis in neonates was defined either as proven or suspected. Proven sepsis was diagnosed when blood and/or cerebrospinal fluid cultures were positive along with the supportive clinical and laboratory findings. In brief, the clinical signs considered indicative of sepsis were: poor peripheral circulation, respiratory distress, cyanosis, irritability, lethargy, apneic spells, tachycardia, bradycardia, unstable temperature, poor feeding, instability of blood sugar levels, and jaundice. Laboratory findings considered indicative of sepsis were: neutrophilia (polymorphonuclear leukocytes $>9,000 / \mathrm{mm}^{3}$ ) or neutropenia (polymorphonuclear leukocytes $<1,000 / \mathrm{mm}^{3}$ ), increased immature to total neutrophil ratio ( $\mathrm{I} / \mathrm{T}>0.2)$, increased $\mathrm{C}$-reactive protein (CRP $>15 \mathrm{mg} / \mathrm{dl}$ ), thrombocytopenia (platelet count $<80,000 \mathrm{~mm}^{3}$ ), disseminated intravascular coagulopathy and $\mathrm{x}$-ray findings consistent with pneumonia, respiratory distress syndrome, and pneumothorax $(24,37)$. The diagnosis of suspected sepsis was established when at least three clinical signs and two laboratory findings of infection were present. Activin-A is increased during perinatal asphyxia/hypoxia and intraventricular hemorrhage $(11-14,29,38)$. In addition, maternal activin-A levels are associated with preeclampsia (39) and the use of antidepressants (30). Hence, neonates born to mothers with preeclampsia or using antidepressants were excluded from our study. Moreover, neonates with asphyxia/hypoxia, intraventricular hemorrhage, congestive heart failure, intrauterine growth retardation, metabolic disorders, major congenital malformations, inborn errors of metabolism, small for dates, and those who had received immunotherapy were also excluded. The study was approved by the Hospital Research Ethics Committee, and informed written parental consent was obtained before neonates were enrolled into the study. All human investigations were conducted according to the principles expressed in the Declaration of Helsinki.

\section{In Vitro Stimulation of Neonatal Peripheral Blood Leukocytes}

Peripheral blood $(4 \mathrm{ml})$ was obtained from healthy neonates $(\mathrm{BW}>$ 2,800 g; GA >38 wk (37-41); $n=9-10)$, and PBMCs were isolated by Histopaque (Sigma-Aldrich, St Louis, MO). PBMCs were cultured in Roswell Park Memorial Institute medium with GlutaMAX (Invitrogen, Carlsbad, CA) supplemented with $2 \%$ penicillin/streptomycin (SigmaAldrich) at $37^{\circ} \mathrm{C}, 5 \% \mathrm{CO}_{2}$ for $1 \mathrm{~h}$. Nonadherent lymphocytes were collected and cultured for 4,24 , or $48 \mathrm{~h}$ in the presence or absence of PHA (0.1 mg/ml; Sigma-Aldrich), as previously described (40).
Flow cytometry (Coulter Epics XL-MCL; Beckman Coulter, Brea, CA) analysis confirmed that lymphocytes were viable ( $96 \%)$ and actively proliferating following PHA stimulation. The majority of lymphocytes were $\mathrm{CD}^{+} \mathrm{T}$ cells $(70-75 \%)$ and the rest were, mainly, B lymphocytes (30-25\%). We used the strong T-cell mitogen PHA to measure T-cell responses specifically, as PHA does not induce the activation of B lymphocytes, monocytes, or DCs $(17,18)$. Moreover, PHA stimulation recapitulates certain features of viral T-cell stimulation (16).

Monocytes $\left(\mathrm{CD} 14^{+}\right)$were isolated from neonatal PBMCs by negative selection using the monocyte isolation kit II (Miltenyi Biotec, Bergisch Gladbach, Germany). Cell purity was $>92 \%$, as determined by flow cytometry. Isolated monocytes were cultured in Roswell Park Memorial Institute medium with GlutaMAX (Invitrogen) supplemented with $2 \%$ penicillin/streptomycin (Sigma-Aldrich) at $37^{\circ} \mathrm{C}$, $5 \% \mathrm{CO}_{2}$ for 4,24 , or $48 \mathrm{~h}$ in the presence or absence of LPS $(1 \mu \mathrm{g} /$ $\mathrm{ml}$; Sigma-Aldrich), as previously described (22). We used LPS to examine the effects of activin-A on responses mediated by neonatal monocytes, as these cells express high levels of the LPS receptor, tolllike receptor-4, on their surface and respond highly to this stimulus (1,19-21). Moreover, LPS stimulation closely resembles monocyte activation with bacterial stimuli (1).

In certain experiments, human recombinant activin-A ( $\mathrm{r}$-activinA; $50 \mathrm{ng} / \mathrm{ml}$; R\&D Systems, Minneapolis, MN) or control (phosphatebuffered saline) were added to the lymphocyte and monocyte cultures during PHA and LPS stimulation. The optimal doses of LPS, PHA, and $\mathrm{r}$-activin-A were determined by dose-response studies (data not shown). A similar dose $(50 \mathrm{ng} / \mathrm{ml})$ of activin-A has been used in several studies for the examination of responses mediated by immune cells $(35,41,42)$.

\section{Cytokine and Chemokine Analysis}

Peripheral blood samples were collected from septicemic neonates on the first, third, and fifth days postinfection or from healthy controls. Serum was obtained after centrifugation at $1,000 \mathrm{~g}$ for $5 \mathrm{~min}$ and stored at $-20{ }^{\circ} \mathrm{C}$ until further analysis. CXCL8 and activin-A levels were measured in the serum by ELISA according to the manufacturer's instructions (Quantikine; R\&D Systems). Activin-A was also measured in the supernatants of PHA- or LPS-stimulated lymphocytes and monocytes at the indicated time points. The lowest detection limits were: $31.2 \mathrm{pg} / \mathrm{ml}$ for CXCL8 and $125 \mathrm{pg} / \mathrm{ml}$ for activin-A. IL-6, IL-10, CXCL8, TNF- $\alpha$, and IL-1 $\beta$ levels were examined in cell culture supernatants by the cytometric Bead Array Flex system (BD Biosciences, San Jose, CA).

\section{In Vitro Analysis of Lymphocyte Proliferation}

Neonatal peripheral blood lymphocytes were cultured into 96-U-bottom tissue culture plates at a density of $2 \times 10^{5}$ cells $/ \mathrm{ml}$ in Roswell Park Memorial Institute medium 1640 and $10 \%$ fetal calf serum (Invitrogen) in the presence of PHA $(0.1 \mathrm{mg} / \mathrm{ml})$ for $24 \mathrm{~h}$. Activin-A $(50 \mathrm{ng} / \mathrm{ml})$ or control (phosphate-buffered saline) were added to the cell cultures. Viable cells were stained with 3-4,5-dimethylthiazol-2-yl-2,5 diphenyl tetrazolium bromide, (Sigma-Aldrich) for $4 \mathrm{~h}$. Medium was removed and the formazan crystal was dissolved by adding $200 \mu \mathrm{l}$ of DMSO (Sigma-Aldrich). Absorbance (at $570 \mathrm{~nm}$ ) was used to measure cell proliferation, as previously described (43).

\section{Statistical Analysis}

Data are presented as mean \pm SEM, unless otherwise mentioned, and were checked for normal distribution by means of the Lilliefors modification of the Kolmogorov-Smirnov test. Paired data at different time points shown in Figures 1 and 3 were analyzed by repeated measures two-way ANOVA (mixed model), followed by Bonferoni post hoc analysis. Kinetics data regarding activin-A (Figure 2a) and CXCL8 (Figure 2b) were analyzed by repeated measures one-way ANOVA (Friedman test), followed by Dunn's post hoc analysis for all pairs. The correlation data between activin-A and CXCL8 concentration at different time points shown in Figure 2c were analyzed by repeated measures two-way ANOVA (mixed model), followed by Bonferoni post hoc analysis and nonparametric Spearman correlation. Data shown in Figures 4 and 5 were analyzed by one-way ANOVA (Kruskal-Wallis test), followed by Dunn's post hoc analysis for all pairs. Statistical 
analysis was performed using statistical package software (GraphPad Prism version 5.03, GraphPad Software, La Jolla, CA). A $P$ value of $<0.05$ was considered statistically significant.

\section{ACKNOWLEDGMENTS}

We are grateful to Dr. Sarafidou for help in the statistical analysis of the data. Our coauthor Marietta Xanthou passed away on 3 September 2013.

\section{STATEMENT OF FINANCIAL SUPPORT}

This work was supported by a research grant obtained from Aghia Sophia Children's Hospital.

Disclosure: The authors state that there is no conflict of interest that could be perceived to bias this work.

\section{REFERENCES}

1. Levy O. Innate immunity of the newborn: basic mechanisms and clinical correlates. Nat Rev Immunol 2007;7:379-90.

2. Lam HS, Ng PC. Biochemical markers of neonatal sepsis. Pathology 2008; $40: 141-8$.

3. Ng PC, Li K, Wong RP, et al. Proinflammatory and anti-inflammatory cytokine responses in preterm infants with systemic infections. Arch Dis Child Fetal Neonatal Ed 2003;88:F209-13.

4. de Kretser DM, O'Hehir RE, Hardy CL, Hedger MP. The roles of activin A and its binding protein, follistatin, in inflammation and tissue repair. Mol Cell Endocrinol 2012;359:101-6.

5. Kariyawasam HH, Semitekolou M, Robinson DS, Xanthou G. Activin-A: a novel critical regulator of allergic asthma. Clin Exp Allergy 2011;41:1505-14.

6. Jones KL, Brauman JN, Groome NP, de Kretser DM, Phillips DJ. Activin A release into the circulation is an early event in systemic inflammation and precedes the release of follistatin. Endocrinology 2000;141:1905-8.

7. Jones KL, de Kretser DM, Clarke IJ, Scheerlinck JP, Phillips DJ. Characterisation of the rapid release of activin A following acute lipopolysaccharide challenge in the ewe. J Endocrinol 2004;182:69-80.

8. Jones KL, Mansell A, Patella S, et al. Activin A is a critical component of the inflammatory response, and its binding protein, follistatin, reduces mortality in endotoxemia. Proc Natl Acad Sci USA 2007;104:16239-44.

9. Morpurgo PS, Cetin I, Borgato S, et al. Circulating levels of inhibin A, inhibin $\mathrm{B}$ and activin $\mathrm{A}$ in normal and intrauterine growth restricted (IUGR) fetuses. Eur J Obstet Gynecol Reprod Biol 2004;117:38-44.

10. Mylonas I, Schiessl B, Jeschke U, et al. Expression of inhibin/activin subunits alpha (-alpha), beta A (-beta (A)) and beta B (-beta (B)) in placental tissue of normal and intrauterine growth restricted (IUGR) pregnancies. J Mol Histol 2006;37:43-52.

11. Florio P, Perrone S, Luisi S, et al. Activin a plasma levels at birth: an index of fetal hypoxia in preterm newborn. Pediatr Res 2003;54:696-700.

12. Florio P, Luisi S, Bruschettini M, et al. Cerebrospinal fluid activin a measurement in asphyxiated full-term newborns predicts hypoxic ischemic encephalopathy. Clin Chem 2004;50:2386-9.

13. Bracci R, Perrone S, Buonocore G. The timing of neonatal brain damage. Biol Neonate 2006;90:145-55.

14. Florio P, Abella R, Marinoni E, et al. Biochemical markers of perinatal brain damage. Front Biosci (Schol Ed) 2010;2:47-72.

15. Rosenberg VA, Buhimschi IA, Dulay AT, et al. Modulation of amniotic fluid activin-a and inhibin-a in women with preterm premature rupture of the membranes and infection-induced preterm birth. Am J Reprod Immunol 2012;67:122-31.

16. Ennis FA, Meager A. Immune interferon produced to high levels by antigenic stimulation of human lymphocytes with influenza virus. J Exp Med 1981;154:1279-89.

17. Greaves M, Janossy G, Doenhoff M. Selective triggering of human T and B lymphocytes in vitro by polyclonal mitogens. J Exp Med 1974;140:1-18.

18. Stentz FB, Kitabchi AE. Activated T lymphocytes in Type 2 diabetes: implications from in vitro studies. Curr Drug Targets 2003;4:493-503.

19. Angelone DF, Wessels MR, Coughlin M, et al. Innate immunity of the human newborn is polarized toward a high ratio of IL-6/TNF-alpha production in vitro and in vivo. Pediatr Res 2006;60:205-9.

20. O’Hare FM, William Watson R, Molloy EJ. Toll-like receptors in Neonatal Sepsis. Acta Paediatr 2013;102:572-8.
21. Yerkovich ST, Wikström ME, Suriyaarachchi D, Prescott SL, Upham JW, Holt PG. Postnatal development of monocyte cytokine responses to bacterial lipopolysaccharide. Pediatr Res 2007;62:547-52.

22. Ebert S, Phillips DJ, Jenzewski P, Nau R, O'Connor AE, Michel U. Activin A concentrations in human cerebrospinal fluid are age-dependent and elevated in meningitis. J Neurol Sci 2006;250:50-7.

23. Ebert S, Zeretzke M, Nau R, Michel U. Microglial cells and peritoneal macrophages release activin A upon stimulation with Toll-like receptor agonists. Neurosci Lett 2007;413:241-4.

24. Ng PC, Lam HS. Biomarkers for late-onset neonatal sepsis: cytokines and beyond. Clin Perinatol 2010;37:599-610.

25. Zhao J, Kim KD, Yang X, Auh S, Fu YX, Tang H. Hyper innate responses in neonates lead to increased morbidity and mortality after infection. Proc Natl Acad Sci USA 2008;105:7528-33.

26. Smith C, Yndestad A, Halvorsen B, et al. Potential anti-inflammatory role of activin A in acute coronary syndromes. J Am Coll Cardiol 2004;44:36975.

27. Sanjabi S, Zenewicz LA, Kamanaka M, Flavell RA. Anti-inflammatory and pro-inflammatory roles of TGF-beta, IL-10, and IL-22 in immunity and autoimmunity. Curr Opin Pharmacol 2009;9:447-53.

28. Michel U, Ebert S, Phillips D, Nau R. Serum concentrations of activin and follistatin are elevated and run in parallel in patients with septicemia. Eur J Endocrinol 2003;148:559-64.

29. Florio P, Perrone S, Luisi S, et al. Increased plasma concentrations of activin a predict intraventricular hemorrhage in preterm newborns. Clin Chem 2006;52:1516-21.

30. Bellissima V, Visser GH, Ververs TF, et al. Antenatal maternal antidepressants drugs affect Activin A concentrations in maternal blood, in amniotic fluid and in fetal cord blood. J Matern Fetal Neonatal Med 2011;24:Suppl 2:31-4.

31. Yndestad A, Ueland T, Øie E, et al. Elevated levels of activin A in heart failure: potential role in myocardial remodeling. Circulation 2004;109:137985.

32. Robson NC, Phillips DJ, McAlpine T, et al. Activin-A: a novel dendritic cell-derived cytokine that potently attenuates CD40 ligand-specific cytokine and chemokine production. Blood 2008;111:2733-43.

33. Chen $\mathrm{Y}, \mathrm{Wu} \mathrm{H}$, Winnall WR, et al. Tumour necrosis factor-a stimulates human neutrophils to release preformed activin A. Immunol Cell Biol 2011;89:889-96.

34. Nüsing RM, Barsig J. Induction of prostanoid, nitric oxide, and cytokine formation in rat bone marrow derived macrophages by activin $\mathrm{A}$. Br J Pharmacol 1999;127:919-26.

35. Semitekolou M, Alissafi T, Aggelakopoulou M, et al. Activin-A induces regulatory $\mathrm{T}$ cells that suppress $\mathrm{T}$ helper cell immune responses and protect from allergic airway disease. J Exp Med 2009;206:1769-85.

36. Mukerji SS, Katsman EA, Wilber C, Haner NA, Selman WR, Hall AK. Activin is a neuronal survival factor that is rapidly increased after transient cerebral ischemia and hypoxia in mice. J Cereb Blood Flow Metab 2007;27:1161-72.

37. Wynn J, Cornell TT, Wong HR, Shanley TP, Wheeler DS. The host response to sepsis and developmental impact. Pediatrics 2010;125:1031-41.

38. Fiala M, Baumert M, Walencka Z, Paprotny M. Umbilical activin A concentration as an early marker of perinatal hypoxia. J Matern Fetal Neonatal Med 2012;25:2098-101.

39. Jacquemyn Y, Zemtsova O. Risk factors and prediction of preeclampsia. Acta Clin Belg 2010;65:1-12.

40. Levy E, Xanthou G, Petrakou E, et al. Distinct roles of TLR4 and CD14 in LPS-induced inflammatory responses of neonates. Pediatr Res 2009;66:179-84.

41. Karagiannidis C, Hense G, Martin C, et al. Activin A is an acute allergenresponsive cytokine and provides a link to TGF-beta-mediated airway remodeling in asthma. J Allergy Clin Immunol 2006;117:111-8.

42. Kariyawasam HH, Pegorier S, Barkans J, et al. Activin and transforming growth factor-beta signaling pathways are activated after allergen challenge in mild asthma. J Allergy Clin Immunol 2009;124: 454-62.

43. Mosmann T. Rapid colorimetric assay for cellular growth and survival: application to proliferation and cytotoxicity assays. J Immunol Methods 1983;65:55-63. 